\title{
Strengthened research ethics, including patient anonymity and informed consent, in MEDLINE and PubMed Central journals
}

\author{
Sun Huh \\ Department of Parasitology and Institute of Medical Education, Hallym University College of Medicine, Chuncheon, Korea
}

Recently, a re-evaluation of two journals from Korea indexed in PubMed Central (PMC) for their ethical and scientific integrity was conducted by the staff of the United States National Library of Medicine (NLM).

The first case dealt with an ethical issue involving Archives of Plastic Surgery, published by the Korean Society of Plastic and Reconstructive Surgeons. The editor of Archives of Plastic Surgery received an email from PMC staff on September 1, 2017 stating that NLM staff had recently identified a number of concerns about the enforcement of ethics policies in Archives of Plastic Surgery, as well as concerns about whether the journal adhered to its posted policies regarding human and animal rights based on a sampling of articles. The editor replied that he would recheck those articles. On October 20, 2017, a notice was sent announcing that the journal would be re-evaluated to assess its adherence to the scientific quality standards of PMC over the course of a 12-week period starting at the end of October; furthermore, it was announced that PMC XML uploading would be temporarily put on hold during the review period. Subsequently, the editorial office of the journal confirmed these ethical issues indicated by the NLM staff and published corrigenda on October 24, 2018. Corrections were issued for eight articles, redescribing Institutional Review Board approval, the provision of informed consent, and permission from the Animal Ethics Committee [1]. Furthermore, an editorial on ethical

\section{Correspondence: Sun Huh}

Department of Parasitology and Institute of Medical Education, Hallym University College of Medicine, 1 Hallimdaehak-gil, Chuncheon 24252, Korea

E-mail: shuh@hallym.ac.kr

Received September 23, 2018 / Revised October 2, 2018 / Accepted October 2, 2018 issues involving animal experiments and identifiable photographs was published on October 30, 2017 to explain the background of the corrigenda [2]. The re-evaluation by the NLM of the journal's ethical and scientific integrity was resolved on December 16, 2017 with the comment that PMC journals should meet the NLM's quality standards [3] and deposition of XML files to PMC was allowed again.

The second case dealt with ethical issues involving another PMC journal published by a scholarly society in Korea. The editor of the journal received an email in May 2018 from PMC expressing concern about the incomplete anonymization of images of patients with or without informed consent. After receiving a response from the editor, PMC notified the journal in July 2019 that it would be re-evaluated for 12 weeks. The editor of the journal published an erratum to correct a case report that included an image of a patient's face in which the eyes were masked, but without informed consent. The second case is still under review, so the results of this process will be of interest for other editors in Korea.

Publishing images of the face is an essential component of articles in certain fields, especially aesthetic surgery, dermatology, otorhinolaryngology, and ophthalmology. Although the patient was anonymized to some extent by masking the eye region, the image could be identified by the patient herself or himself, as well as by his or her family. Furthermore, other parts of the body besides the face can be identified by the patient or his or her family. Therefore, the author of an article including a photo of any part of a patient's body is recommended to obtained informed consent [4]. 
A common characteristic of the above two journals is that those ethical issues were screened during their application to MEDLINE. The United States National Medical Library Literature Selection Technical Review Committee (LSTRC) checks applying journals meticulously. The ethics policy and whether the journal follows that policy are essential components of the many items that are reviewed. For example, another PMC journal received the following feedback after its application to MEDLINE on June 23, 2016: "Lax ethics practice, e.g., a case report showed a barely disguised full facial photograph without an ethics statement (e.g., patient's consent). There is some inconsistency in the following of these ethical guidelines (written consent and IRB statements are lacking in a few articles)" [5]. Although this ethical issue was raised, the results were not transmitted to the PMC staff at that time. Therefore, we can surmise that ethical issues raised during the MEDLINE application process began to be shared with PMC starting in 2017.

For local biomedical journals written in English only, the best way to survive in the jungle of the competitive scholarly journal market is to be searchable in PubMed, which is maintained by the NLM although the power of Google Scholar search has soared recently. There are two ways to be a PubMed journal: first, being indexed in MEDLINE; second, becoming a PMC journal. Therefore, all English-language biomedical journals published in Korea have tried to become PMC journals and to be indexed in MEDLINE. Journals from Korea started to become PMC journals in 2008. Ten years later, the number of PMC journals from Korea was 116, excluding duplicate titles. If PMC journals are limited to those published by societies or institutes and to those with immediate free access and deposition of all articles, Korea is the first-ranking country [5]. However, the number of MEDLINE journals from Korea was 29 as of 2018. This means that the barrier to become a MEDLINE journal is higher than the barrier to become a PMC journal. Starting in 2016, some journals from Korea began to be rejected during the PMC application process. Therefore, we can see that the PMC review criteria have also become more stringent, reaching the level of the MEDLINE review process. One reason for rejection by PMC is a journal's compliance with the Principles of Transparency and Best Practice in Scholarly Publishing [6]. PMC's policy statement on scientific, editorial, and technical standards (reevaluation) announced that "if a publisher is found to not be following established industry best practices, NLM will cease collecting the publisher's journals and not accept applications for any of the NLM Literature databases, in- cluding PMC and MEDLINE, for a minimum of three years" [3].

Many biomedical journals from Korea, including Archives of Craniofacial Surgery became internationalized through being searchable in PubMed as a PMC or MEDLINE journal. Removal from PMC or MEDLINE for 3 years would pose a critical issue for a journal's reputation; furthermore, removal from PMC or MEDLINE would make it challenging to solicit manuscripts from all over the world. Therefore, for survival as a local society journal, editors should do their best to adhere to the Principles of Transparency and Best Practice in Scholarly Publishing, including research and publication ethics. Informed consent, institutional review board approval, and/or animal right statements should be checked meticulously if applicable. Adherence to ethical issues is the foundation of editing journals in a way that ensures scientific and ethical integrity.

\section{CONFLICT OF INTEREST}

No potential conflict of interest relevant to this article was reported.

\section{REFERENCES}

1. Editorial Office, Archives of Plastic Surgery. Corrigenda: omission of the description of informed consent on the identifiable photos and the description on ethical treatment of experimental animals. Arch Plast Surg 2017;44:575-6.

2. Huh S. How to deal with ethical issues involving animal experiments and identifiable photographs in articles published in Archives of Plastic Surgery. Arch Plast Surg 2017;44:475-6.

3. National Center for Biotechnology Information. PMC policies [Internet]. Bethesda, MD: U.S. National Library of Medicine; 2013 [cited 2018 Sep 23]. Available from: https://www.ncbi. nlm.nih.gov/pmc/about/guidelines/\#standards.

4. Huh S. Ethical issue in preparing manuscript on esthetic patients. Arch Aesthetic Plast Surg 2014;20:1-2.

5. Huh S. How to prepare Endocrinology and Metabolism for reapplication to MEDLINE. Endocrinol Metab (Seoul) 2017;32: 58-61.

6. Huh S. Adherence of the Annals of Pediatric Endocrinology and Metabolism to the Principles of Transparency and Best Practice in scholarly publishing. Ann Pediatr Endocrinol Metab 2018;23:1-3. 